\title{
The XIth International Online Symposium on Heat Shock Proteins in Biology and Medicine
}

\author{
Organizers: Stuart K. Calderwood, Elizabeth Repasky \& Len Neckers \\ October 27-29, 2021
}

IN MEMORIAM: Claudina Rodrigues-Pousada (1941-2021)

Stuart K. Calderwood ${ }^{1} \cdot$ Elizabeth Repasky ${ }^{2} \cdot$ Len Neckers $^{3} \cdot$ Lawrence Hightower $^{4}$

Published online: 20 January 2022

(c) Cell Stress Society International 2021

\begin{abstract}
Single cell and multicellular organisms encounter physical stress from their environment as well as behavioral stress experienced in more complex organisms. As these stresses can present an existential threat, organisms respond with a coordinated response at the tissue and cellular level, the heat shock response (HSR) and this was the major theme of the symposium. Much of the meeting was concentrated on the heat shock proteins (HSPs), the effector molecules of the response. The balance between the potency of the HSR and the experience of stress naturally plays a key role in the etiology of many disease. Roles in cancer, the immune response, cell metabolism and aging were discussed at length at the meeting. Finally, a major goal of this field is to enhance the HSR in pathological conditions where it becomes inadequate or over stimulated and important findings regarding pharmacological approaches to modulating the HSR were discussed.
\end{abstract}

The symposium consisted of twenty-five presentations, including one keynote address and seven symposia. There were over 200 participants representing 23 countries attending this virtual meeting. Professor John T. Lis was awarded the CSSI Medallion for Career Achievement. Senior Fellow Dr Charles Giardina and Dr Leighton Core, both Lis laboratory graduates and faculty colleagues at the University of Connecticut, helped introduce Professor Lis.

Stuart K. Calderwood

scalderw@bidmc.harvard.edu

1 Beth Israel Deaconess Medical Center, Harvard Medical School, Boston, MA, USA

2 Roswell Park Comprehensive Cancer Center, Buffalo, NY, USA

3 NCI/NIH, Bethesda, MA, USA

4 Department of Molecular and Cell Biology, University of Connecticut, Storrs, CT, USA
The Keynote Speech was given by: Professor John T. Lis, Cornell University.

What the heat shock system has taught us about transcription regulation

The most immediate and comprehensive regulator of the HSR is the transcription factor HSF1, which regulates expression of all the major HSP genes in eukaryotes. Dr Lis described his key role in the evolution of the understanding of HSF1 regulation, ranging from the discovery of response elements in $H S P$ genes to the complex regulation of RNA polymerase II which permits controlled and modulated transcription. Dr Lis showed how understanding of HSF1 has also allowed a more general appreciation of the mechanisms of transcription in the majority of eukaryotic genes. He described his role in the development of powerful methods such as ChIP-sequence and Gro-Seq that have been instrumental in the study of a wide spectrum of genes simultaneously. The symposium attendees were thus able to appreciate the steps in evolution of understanding of 
transcription through the lens offered by Dr Lis' studies of HSF1 and the HSR.

\section{Symposium 1: HSPs in Cancer}

Chair, Mehdi Mollapour, State University of New York, Syracuse, NY.

It has become clear that HSF1 and the HSPs play key enabling roles in cancer, and this session addressed some of the mechanisms involved.

\section{The 2021 Susan Lindquist Memorial Lecture}

Ruth Scherz-Shouval, Weizmann Institute of Science, Rehovot, Israel.

Stress and mutational dependencies shaping the tumor microenvironment.

Dr Scherz-Shouval described her exciting studies on the role of HSF1 in tumorigenic properties of cancer-associated fibroblasts (CAF). It has emerged that CAF provide much of the driving force for cancer development in terms of materials secreted into the extracellular microenvironment (ECM) and that HSF1 plays a fundamental role in this regard.

In a remarkable convergence, the studies of Stuart Calderwood, Harvard Medical School, Hsp72 is essential for mammary cancer metastasis and regulates ECM gene expression, showed a similar role for the inducible Hsp70 genes (HSPA1A, $H S P A 1 B$, jointly described as Hsp72) in mammary cancer. He demonstrated that ECM expression and CAF are dependent on Hsp72, and loss of Hsp72 led to drastically reduced metastasis. We gleaned from both presentations that the HSR thus seems to play a promoting role in cancer.

\section{Symposium 2: HSP inhibitors and disease treatment, HSP therapies}

Chairs, Len Neckers, NCI/NIH Bethesda, Giorgio Colombo, University of Pavia.

HSP dysregulation occurs in many disease types, and this session was devoted to asking whether chemical inhibitors could address some of the pathology.

Heath Ecroyd, University of Wollongong, Australia.

Uncovering new aspects of the molecular chaperone action of DNAJB proteins.
Dr Ecroyd described an elegant method for the study of protein aggregation and cellular inclusions that involved exploiting a destabilized isoform of firefly luciferase (R188Q/R261Q Fluc; termed FlucDM) and quantitative flow cytometry. The experiments suggested that $\mathbf{J}$ domain proteins DNAJB6 and DNAJB8 possess two distinct regions for binding substrates, one that is responsible for binding $\beta$-hairpins that form during amyloid formation and another that interacts with exposed hydrophobic patches in aggregation-prone clients.

Jason Gestwicki, University of California San Francisco.

Screening for chaperone vulnerabilities in prostate cancer.

Prostate cancer is known to be driven by androgen receptor (AR) signaling. The stability and function of AR are controlled by molecular chaperones, including Hsp70 and Hsp90. However, there are $\sim 130$ additional chaperones. Using functional genomics and synthetic lethal screens, the investigators have identified additional chaperones, including Hsp60, that may provide selective vulnerabilities in advanced prostate cancer, indicating that a broad network of chaperones maintains the oncogenic phenotype in prostate cancer.

Jane Trepel, NCI/NIH, Bethesda, MD.

Update on HSP90 inhibitors in oncology clinical trials.

Dr Trepel's talk gave an illuminating update on HSP90 inhibitors in current oncology clinical trials, including therapies that incorporate HSP90-targeted drug conjugates. Hsp90 inhibitors of both canonical and non-canonical types were discussed.

In a slight departure, Joachim Clos, Bernhard Nocht Institute for Tropical Medicine, Hamburg, Germany, spoke about: The Leishmania donovani HSP90 and its impact on stage-specific protein synthesis and chromatin structure. Leishmania donovani is a human-pathogenic parasite of the subtropics and causes a fatal infection in humans. Dr Clos showed that HSP90 is a substrate in Leishmania for at least two signal transduction pathways. This suggests a role as effector for protein kinases that plays a pivotal part in the parasite's life cycle control, as its inhibition causes heterochromatin formation at transcription start regions, mimicking the effects of stage differentiation. The data suggested novel levels of regulation for Hsp90.

Overall, the symposium highlighted the ongoing advances in the field for Hsp90 drugs and new approaches to targeting the chaperone network. 


\section{Symposium 3: Extracellular chaperones}

Chair Dimitra Bourboulia, State University of New York, Syracuse, NY.

This symposium explored the extrinsic and extracellular features of the HSR that extend the range of the response to cell-cell and inter-tissue interactions.

Patricija van Oosten-Hawle, University of Leeds, UK.

Regulation of inter-tissue stress responses in an organism.

This presentation dealt with transcellular chaperone signaling (TCS), an inter-tissue signaling mechanism activated upon tissue-specific alterations of the essential molecular chaperone Hsp90. This leads to the cell non-autonomous upregulation of protective chaperones at an organismal level that can benefit an animal's health span and stress resistance, activation of chaperone expression and its effects on animal behavior.

Wei Li, University of Southern California.

Secreted/extracellular Hsp90: secretion, action and function during wound healing and cancer progression.

$\mathrm{Dr} \mathrm{Li}$ addressed another aspect of the extracellular features of the HSR, the physical release of Hsp90 and its binding to target cells, triggering wound healing in damaged skin epithelium and stimulating tumor cell migration. Dr Li described how Hsp90 was released in extracellular vesicles and as free protein, and could bind to the LRP1 receptor, triggering intracellular signaling and regulatory effects in receptor cells.

Michael Lynes, University of Connecticut.

Metallothionein: an intracellular stress response protein with pro-inflammatory extracellular activities.

Dr Lynes discussed another example of the deployment of intracellular molecules in inter-tissue signaling, involving the metal regulatory molecule metallothionein MT. He showed that extracellular MT can alter chemotactic cell movement and augment lymphoproliferation. These fundamental changes appear to exacerbate several different forms of chronic inflammation.

Ed O'Brien, University of Calgary Cumming School of Medicine.

Heat shock protein 27 immune complex altered signaling and transport (ICAST).
Vaccination of mice with rHSP25 (murine ortholog of human rHSP27) increased anti-HSP25 antibody levels, attenuated atherogenesis, and reduced systemic and hepatic inflammation. The powerful role of Hsp27-anibody complexes in mediating this response was discussed.

Yongzhang Luo, Tsinghua University, Beijing, China.

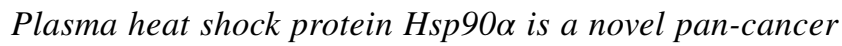
biomarker: from basic research to clinical medicine

Dr Luo followed up on reports from his lab that Hsp90 $\alpha$ secretion by tumor cells, and the level of plasma Hsp90 $\alpha$ are positively correlated with tumor malignancy in cancer patients. He reported here the development of a quantitative detection kit to measure plasma Hsp90 $\alpha$ based on ELISA, and clinical trials demonstrating that plasma Hsp90 $\alpha$ could be a novel pan-cancer biomarker.

The symposium showed the growing significance of the extracellular component of the HSR.

\section{Symposium 4: Responses to changing environments}

Chairs Melody Clark, British Antarctic Survey and Matthias Mayer, University of Heidelberg.

It seems indisputable that the world environment is changing rapidly, and this symposium attempted to address homeostatic mechanisms that organisms might use to counter such external influences.

Manuela Truebano, University of Plymouth, UK.

\section{Mechanisms of cross-tolerance in aquatic invertebrates}

Marine ectotherms are increasingly threatened by multiple anthropogenic stressors, which can occur both in and out of phase with each other, particularly in highly variable intertidal environments. The interaction between two stressors acting sequentially can lead to a reduced physiological impact of the second stressor, a phenomenon known as 'cross-tolerance'. Indeed, Dr Truebano showed that thermal acclimation could lead to hypoxia tolerance, which however did not seem to involve HSP induction.

Peter Lund, University of Birmingham, UK.

The role of a non-essential chaperonin protein in Mycobacteria 
Dr Lund described the role of the non-essential chaperonin (Cpn60.1) in the fish bacterial pathogen Mycobacterium marinum, as it has previously been shown to play a part in the pathogenesis of the closely related M. tuberculosis, the causative agent of TB in humans. His data indicated that this chaperonin requires phosphorylation in order to become active as a chaperone, although its role in pathogenesis does not require its chaperone activity.

Adam Reitzel, University of North Carolina Charlotte.

Phylogenetic diversity and expression dynamics of HSP70s in the estuarine cnidarian Nematostella vectensis.

Dr Reitzel described the phylogenetic diversity of HSPs from the starlet sea anemone Nematostella vectensis as well as their transcriptional variation. Expression of the five HSP70s varied significantly during development as well as among different cell types. In response to temperature shock and metal exposure, about half of the HSPs showed increased expression, with treatment-specific variation in HSP induction.

Rochelle Buffenstein, Calico Life Sciences, LLC.

Pristine proteostasis in naked mole-rats.

The naked mole-rat (Heterocephalus glaber) shows few agerelated changes in composition and physiology over its four decades of life, providing a counterexample to the inevitability of mammalian aging. What sets these mouse-sized rodents apart from humans, mice, and many other animals, are findings suggesting that there is, in fact, no age-dependent decline in the abundance or function of the various proteostasis network components. Indeed, throughout their long lives, there are signs of well-maintained, albeit reduced rates of translation, high levels of molecular chaperones, and increased abundance and activity of the protein degradation machinery. As a result, unlike in other species, there is minimal build-up of polyubiquitinated proteins or oxidized proteins with age.

\section{Symposium 5: Molecular chaperones and the immune response}

Chair, Lea Sistonen, Abo Akademi University, Turku, Finland.

Sharon Evans, Roswell Park Comprehensive Cancer Center, Buffalo, NY.

Thermal regulation of the immune landscape in the tumor microenvironment.
Dr Evans has shown that mild heat shock can profoundly alter the penetration of $\mathrm{T}$ lymphocytes into tumors increasing the effectiveness of immunotherapy. These effects involved the remodeling of the IL-6 receptor system within the tumor vascular epithelium. In these studies, it was shown for the first time that heat can also reduce Treg penetration into tumors and increase CD8+/Treg ratios, also in an IL6dependent manner.

Mauricio Rigo, Rice University, Houston, TX, on behalf of Cristina Bonorino, UFCSPA, Porto Alegre, Brazil.

Post-translational modifications of the host expression system modulate Hsp70 activity.

HSP orthologs from different species often have different effects on immune and inflammatory responses. Dr Rigo described studies on Hsp70 PTMs that may differ in various species and illustrated their influence on the resulting immune responses, many of which had previously been puzzling.

Shawn X-Y Wang, Virginia Commonwealth University, Richmond, VA.

Use of a chaperone-based immunostimulatory agent to reshape the tumor microenvironment for promoting anticancer immunity.

Dr Wang presented data on HSP-based tumor immunotherapy approaches, utilizing a construct containing flagrp 170 built on the backbone of the glucose-regulated protein 170 . This molecule is thus equipped with a highly immunostimulatory pathogen-associated "danger" signal while retaining a potent antigen cross-presenting activity for optimal $\mathrm{T}$ cell activation. This agent creates an immune-inflamed tumor phenotype, indicated by induction of a set of cytokines in the TME known to support antitumor immunity while retaining antigen carrying properties.

Elizabeth Repasky, Roswell Park Comprehensive Cancer Center, Buffalo, NY.

How does stress suppress antitumor immunity.

This presentation highlighted the role of chronic organismal stress in tumor immunity. Tumors require adrenergic innervation which appears to have an immunosuppressive influence. The investigators showed that inhibiting stress signals with beta blockers could increase $\mathrm{T}$ cell levels and $\mathrm{T}$ cell signaling in tumors and reduce function of immunosuppressive MDSC. The significance of this work was highlighted 
by a study showing that the burden of financial stress significantly lowered patients' responses to radiotherapy.

\section{Symposium 6: Chaperone networks and dysregulation in disease}

Chair, Andrew Truman, University of North Carolina Charlotte, Charlotte, NC.

It has become clear that chaperones exist in complexes within cells and here this symposium aimed to address the significance of such networks.

Gabriela Chiosis, Memorial Sloan Kettering Cancer Center, New York, NY.

Epichaperomes - on how to impact and rebalance proteomewide protein-protein interaction networks in disease.

Dr Chiosis described how chaperones and co-chaperones become remodeled in stress and disease into complex, longlived oligomeric structures known as epichaperomes. These structures act as pathologic scaffolds that rewire proteinprotein interactions, and their formation causes thousands of proteins to associate improperly within cells. There appears to be unique spatio-temporal formation of epichaperome structures in individual malignant and neurodegenerative diseases. These structures can be antagonized by targeting key chaperone components that, at critical drug doses, cause the network to collapse.

Yuka Okusha, Beth Israel Deaconess Medical Center, Harvard Medical School.

MicroRNA-570 targets the HSP chaperone network and inhibits breast cancer growth and tumor cell migration.

Dr Okusha described an approach to targeting chaperones using micro-RNA. To bypass the inherent redundancy in the chaperone network, she employed a specific microRNA (mir), showing that mir-570 could silence expression of multiple HSPs and inhibit the heat shock response. The studies also reported reduced tumor growth with mir alone or in combination with cytotoxic drugs. These data suggested this approach as a promising method to target chaperone networks.

Ahmed Chadli, University of Augusta, Augusta, GA.

The Hsp90 co-chaperone UNC45A promotes breast cancer growth through the mitotic kinase NEK7.

The heat shock protein 90 (HSP90) co-chaperone UNC-45A is known to contribute to tumorigenesis. Dr Chadli showed here that UNC45A localizes to the cancer cell nucleus and promotes expression of the mitotic kinase NIMA-related kinase 7 (NEK7) through upregulation of glucocorticoid receptor transcriptional activity. Loss of UNC45A caused disruption of mitotic processes. Consequently, cells lacking UNC45A stalled in metaphase and cytokinesis and ultimately underwent mitotic catastrophe. Thus, UNC45A represents a promising new therapeutic target to inhibit cancer cell growth in solid tumor types.

\section{Symposium 7: Heat shock factors and Molecular Chaperones in Regulation of Metabolism}

Chairs, Brian Freeman, University of Illinois, Urbana-Champaign, IL, and Abhinav Joshi, NCI/NIH Bethesda, MD.

Here, the studies aimed to examine the emerging role of the HSR in intermediary metabolism and pathology arising from such a role.

M. Gabriella Santoro, University of Rome Tor Vergata.

HSF1 and AIRAP: from cancer to coronaviruses.

Dr Santoro described studies identifying the gene encoding AIRAP (arsenite-inducible RNA-associated protein), also known as ZFAND2A (zinc finger AN1-type domain-2a gene), as a novel human heat shock gene whose expression is strictly controlled by HSF1. This group further showed that AIRAP is implicated in the response of human cancer cells to treatment with antineoplastic drugs, including proteasome inhibitors and cyclopentenone prostanoids.

Len Neckers, Urologic Oncology Branch, CCR, NCI/NIH.

Role of Hsp70 in metabolic re-wiring and therapeutic sensitivity of castration-resistant prostate cancer.

Dr Neckers presented data showing that the Hsp70 inhibitor JG98 re-wires mitochondrial metabolism in castration resistant prostate cancer (CRPC) concomitant with down-regulation of oxidative phosphorylation and increased aerobic glycolysis, metabolic properties resembling those in normal prostate. Under these conditions, Hsp70 targeting drug JG98 restored sensitivity to androgen deprivation therapy and phenocopied the impact on CRPC of inhibiting mitochondrial electron chain complex I (with IACS-010759) or complex II (with CNC332). Furthermore, JG98 displayed combinatorial activity with IACS-010759, both in vitro and in vivo. Thus, Hsp70 inhibition is a potential therapeutic strategy to treat CRPC, either as monotherapy or as a sensitizer for other mitochondrial inhibitors. 
Nahid F. Mivechi, Georgia Cancer Center, Augusta University.

HSF 1 controls lipid utilization and energy expenditure: A nexus to the development of obesity and insulin resistance.

Dr Mivechi's group had previously shown that deletion of $h s f l$ from mice significantly suppressed resistance to high-fat diet-induced obesity and insulin resistance. They explored here three highly metabolically active tissues: liver, muscle, or fat to reveal which of these tissues was key for the systemic energy loss observed following $h s f l$-deletion that leads to their resistance to develop obesity and insulin resistance. Their studies indicate that HSF1 deletion from adipocytes led to proton leakage. In addition, $h s f l$ negative mice removed glucose from the bloodstream faster than wild-type mice and metabolized more glucose. The studies have clear implications for a controlling role for HSF1 in metabolism and could suggest significance in Type 2 diabetes and aging.

Acknowledgments We thank Dr Mehdi Mollapour for his role in designing the program and creating the online format of the meeting and Rachael Kim for her expert technical role in coordinating the Zoom presentations and discussions. We are grateful to Drs Patricija van Oosten-Hawle and Andy Truman for help and suggestions on the content of the program. We are also grateful to Helen Neumann for her crucial role in administering the organization of the program.

Publisher's note Springer Nature remains neutral with regard to jurisdictional claims in published maps and institutional affiliations. 